\title{
Enhanced light extraction from emitters close to clusters of resonant plasmonic nanoantennas.
}

\author{
O. L. Muskens, ${ }^{*}$ J. Gómez Rivas \\ FOM Institute for Atomic and Molecular Physics AMOLF, c/o Philips Research \\ Laboratories, High Tech Campus 4, 5656 AE, Eindhoven, The Netherlands
}

\begin{abstract}
We perform time-resolved fluorescence spectroscopy on clusters of plasmonic nanoantennas covered with a dye-polymer mixture. Dimer antenna structures were fabricated consisting of two interacting gold nanorods with varying lengths and interparticle separation. By combining four individual antennas into a cluster within a diffraction limited spot size, we can couple out half of the dye molecule fluorescence via antenna plasmons. Two-dimensional confocal fluorescence lifetime scans visualize the spontaneous emission enhancement of the molecular fluorescence around the antenna clusters.
\end{abstract}

Key words: Plasmon enhanced, Fluorescence, Nanoantennas PACS: 78.67.bf, 42.25.Fx, 73.20.mf

Enhancement of the excitation and emission rates of fluorescent molecules and quantum dots by coupling to well-defined plasmonic nanosystems is currently seeing an enormous activity .[1-7] One can generally find applications in which the local enhancement of an optical pump is important, such as SERS, biosensing, and near-field optics (NSOM). On the other hand, enhancement of spontaneous emission rates via the plasmonic density of states is of importance for the improvement of light-emitting devices. In particular, the improvement of the quantum efficiency via modification of the branching ratio between radiative and nonradiative decay channels leads to more efficient light sources. Enhancement of radiative rates using plasmonic antennas may

* Corresponding author. Address: for Atomic and Molecular Physics AMOLF, P.O.Box 41883, 1009DB, Amsterdam, The Netherlands, Tel.: +31-206081234.

Email address: muskens@amolf.nl (O. L. Muskens,).

URL: http://www.plasmonics.eu (J. Gómez Rivas). 
eventually lead to novel applications in quantum optics, strong coupling, and plasmonic nanolasers.

Here we present confocal fluorescence lifetime imaging experiments of fluorescence emission of a high efficiency dye around resonant metal nanostructures. We use dimer nanoantennas consisting of two adjacent gold nanorods, since these have shown large local field enhancements in the gap between the two nanorods. [8] These local field effects are strongly dependent on both the length of the nanorods and their separation, and are related to standing wave resonances of plasmon polariton modes in the rods. [9] In this study, the plasmon resonance position is tuned to the emission band of the dye molecules, in order to enhance their radiative decay rate and hence the quantum efficiency.

Arrays of nanoantennas have been fabricated using high-resolution e-beam lithography with a $40 \mathrm{kV}$ electron beam lithography system (FEI Company). Figure 1 shows a typical array, consisting of clusters of 4 antenna structures in a small submicrometer area. We have chosen for this configuration of 4 antennas to maximize the amount of dye molecules in a diffraction limited laser spot that will couple to plasmons. In the array, two antenna parameters, namely the antenna arm lengths and gap widths, are varied along the two directions, allowing for a fast exploration of a large parameter space in a single scan. In the SEM image, the metal nanostructures were already covered with a 10-nm layer of silica, which acts as a spacer between the metal and the dye. The first column on the left contains only single nanorods, with the length of the long axis varying from $70 \mathrm{~nm}$ (bottom left) to $150 \mathrm{~nm}$ (top left). The second to eight columns contain structures where the gap between two adjacent nanorods has not opened up; these were designed with too narrow gaps for the e-beam writing. The magnified part of the array shown in Fig. 1(b) shows the transition from interconnected nanorods to dimer antennas with a gap $\Delta$ of $20 \mathrm{~nm}$. Columns 9 to 14 of Fig. 1(a) contain dimers with gap spacings varying from $20 \mathrm{~nm}$ up to $70 \mathrm{~nm}$.

After deposition of the sol-gel silica spacer layer, a 10-nm layer of polymer-dye mixture was spincoated. As a dye we use Atto680 (Attotec GmbH), which is a highly photostable, water soluble dye with specified quantum efficiency in water of $30 \%$, combined with a decay time of $1.8 \mathrm{~ns}$, and values in ethanol of $40 \%$ and $3.4 \mathrm{~ns}$, respectively. The decay time in the spincoated thin-film was measured to be $3.3 \pm 0.1 \mathrm{~ns}$, from which we estimate a corresponding quantum efficiency of $40 \%$.

Fluorescence lifetime imaging (FLIM) was performed using an optical microscope equipped with laser-scanning confocal scanning head (Nikon) synchronized to a commercial picosecond FLIM system (Picoquant). In this configuration, both the pump and the detection foci are scanned synchronously over the sample using a galvanometric scanning mirror pair, as shown schemati- 
cally in Fig. 2.[10] Fluorescence photons are detected, after confocal filtering, by means of a fast silicon avalanche photodetector. The arrival time of the photons is measured using a time-to-amplitude convertor. The confocal scanning microscope is optimized to work at high frame rates, limiting the pixel dwell time per pass to $64 \mu \mathrm{s}$. The FLIM acquisition system continuously synchronizes the arriving photons with the scanning system to allow a 2D image to be built up over subsequent confocal scans. Given the obtained fluorescence count rates of around $20 \mathrm{kcts} / \mathrm{s}$, an integration time of $10 \mathrm{~ms}$ per pixel was sufficient for a lifetime analysis. As a pump we use $\sim 150$ ps pulses from a pulsed diode laser operating at $5 \mathrm{MHz}$. The laser is polarized using a linear polarizer and focused using a $100 \times$, N.A. $=0.9$ microscope objective. Fluorescence photons are collected using the same objective; different polarizations of the emitted light are selected using a polarization filter before the detector. The time resolution is limited by the combination of pump laser and detection electronics to $0.3 \mathrm{~ns}$. We did not use any deconvolution on the fluorescence decay curves, since this would only be reliable at very high signal-to-noise ratios above our current levels.

The collected lifetime images are analyzed using double-exponential decay fits, as shown in Fig. 2. We choose this approach since we found a considerable background of dye molecules in the diffraction limited volume that does not couple to the nanoantenna structures. This background component is modelled as a single-exponential with a variable amplitude $A_{1}$ and fixed decay rate $\gamma_{1}$ of $0.3 \mathrm{~ns}^{-1}$. The additional, faster decay component can be attributed to the fraction of dye molecules that is significantly coupled to the nanoantennas. Note that, in principle, this component consists of an inhomogeneous distribution of decay rates. However, due to the limited resolution, we are unable to resolve this distribution. Therefore, we model the antenna-coupled fraction by a single, average decay component with amplitude $A_{2}$ and decay rate $\gamma_{2}$, as shown in Fig. 2.

Figure 3(a-d) shows the analysis of a fluorescence lifetime image of the array of antenna clusters of Fig. 1, for both excitation and emission polarizations parallel to the antenna long axes. We observe clear effects of the antenna parameters on the enhancement, as we explain below. Figure 3(a) shows the total collected photons $I$, normalized to the intensity averaged over the whole image, denoted by $I_{0}$. Figure. 3(b) shows the fitted amplitude of the background component $A_{1}$, and Fig. $3(\mathrm{c})$ that of the antenna-coupled fraction $A_{2}$, both normalized to the average value of $A_{1}$ over the whole image, denotes as $A_{0}$. The decay rate enhancement of the antenna-coupled fraction $\gamma_{2}$ is shown in Fig. 3(d).

We observe several important effects. Firstly, the amplitude $A_{2}$ of the antennacoupled component shows a strong maximum for each series of separated dimer antennas with fixed gap width (i.e. a single column in Fig. 3(c)). The exact 
length at which the maximum occurs corresponds to the overlap of an antenna resonance with the emission wavelength.[11,12] The condition for resonance at the emission wavelength shifts to smaller antenna lengths with decreasing gap distance. This behavior is associated with a redshift of the plasmonic modes due to capacitive coupling of antenna arms. It has been shown theoretically[9] and experimentally[8] that this redshift is accompanied with a large local field enhancement in the antenna gap. Indeed, we observe an additional enhancement of $A_{2}$ for the antennas with narrow gaps, up to $A_{2} / A_{0}=4$. Associated with the increase in $A_{2}$, we find also a strong enhancement of the fast decay component $\gamma_{2}$ up to 7 times the normal rate $\gamma_{1}$ in absence of antennas. The total intensity $I$ increases to more than $1.4 I_{0}$.

An abrupt transition is observed between the columns containing antennas with interconnected arms (columns 2-8) and those where the arms are well separated (i.e. column 9-14). This can be attributed to a transition from the conductively coupled to capacitively coupled regime, associated with a discontinuity in spectral resonances. [13,14] The monomers in column 1 show the same trend as the dimers with large gap in column 14, indicating that capacitive coupling is negligible for the $70 \mathrm{~nm}$ gap spacing.

The total intensity $I$ shows an increase at the location of the most resonant dimer antennas with narrow gap width $\Delta$. Form the intensity enhancement we can estimate the quantum efficiency assuming the absence of pump enhancement.[12] For the strongest antennas, we find an average quantum efficiency of $56 \pm 4 \%$. From the decrease of the slow background component $A_{1}$ at the location of the antennas in Fig. 3(b) we estimate that roughly half of the dye molecules is significantly coupled to the nanoantennas. Since only this half of the population contributes to the quantum efficiency enhancement, one can estimate that the maximum quantum efficiency of the coupled antennas reaches $72 \%$. This estimate is in agreement with single-quantum dot measurements using a two-dimensional scanning optical antenna probe[7]. Our results demonstrate that enormous improvements will be feasible in a realistic device configuration.

In conclusion, we have demonstrated strong modification of the spontaneous emission of a fluorescent dye by resonant plasmonic nanoantennas. Using confocal fluorescence lifetime imaging, we can map out the effects of individual clusters of nanoantennas. We have analyzed these images using a doubleexponential decay model accounting for a significant fraction of dye molecules that is not coupled to the antennas.

We acknowledge B. Ketelaars and P. Vergeer for technical assistance and V. Giannini and J. Sánchez Gil for stimulating discussions. This work is part of the research program of the "Stichting voor Fundamenteel Onderzoek der Materie (FOM)", which is financially supported by the "Nederlandse Organ- 
isatie voor Wetenschappelijk Onderzoek (NWO)", and is part of an industrial partnership program between Philips and FOM.

\section{References}

[1] E. Ozbay, Science 311, 189 (2006)

[2] P. Anger, P. Bharadwaj, L. Novotny, Phys. Rev. Lett. 96, 113002 (2006)

[3] S. Kühn, U. Hakanson, L. Rogobete, V. Sandoghdar, Phys. Rev. Lett. 97, $017402(2006)$

[4] S. Gerber, F. Reil, U. Hohenester, T. Schlagenhaufen, J.R. Krenn, A. Leitner, Phys.Rev.B 75, 073404 (2007)

[5] J. S. Biteen, N. S. Lewis, H. A. Atwater, H. Mertens, A. Polman, Appl. Phys. Lett. 88, 131109 (2006)

[6] H. Rignault, J. Capoulade, J. Dintinger, J. Wenger, N. Bonod, E. Popov, T. W. Ebbesen, P.-F. Lenne, Phys. Rev. Lett. 95, 117401 (2005)

[7] J. N. Farahani, D. W. Pohl, H.-J. Eisler, B. Hecht, Phys. Rev. Lett. 95017402 (2005)

[8] P. Mühlschlegel, H.-J. Eisler, O. J. F. Martin, B. Hecht, D. W. Pohl, Science 308, 1607 (2005)

[9] J. Aizpurua, G. W. Bryant, L. J. Richter, F. J. García de Abajo, B. K. Kelley, T. Mallouk, Phys. Rev. B 71, 235420 (2005)

[10] J.R. Lakowicz, Principles of fluorescence spectroscopy (Kluwer Academic, New York, 1999)

[11] O. L. Muskens, V. Giannini, J. A. Sánchez Gil, J. Gómez Rivas, cond.mat./0612689 (2006).

[12] O. L. Muskens, V. Giannini, J. A. Sánchez Gil, J. Gómez Rivas, Nano Lett. 6, $552(2006)$

[13] T. Atay, J.-H. Song, and A. V. Nurmikko, Nano Lett. 4, 1627 (2004).

[14] I. Romero, J. Aizpurua, G. W. Bryant, and F. J. García de Abajo, Opt. Express 14, 9988 (2006). 


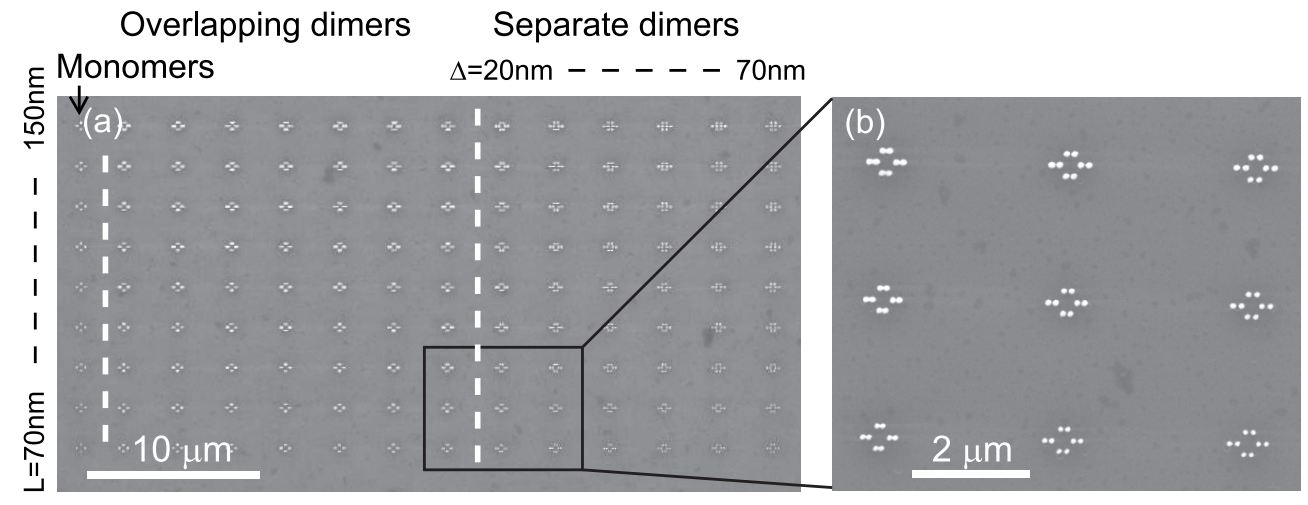

Fig. 1. Figure 1 SEM image of a nanoantenna array with varied antenna arm lengths (vertical) and antenna gaps (horizontal), with detailed images of several single nanorods and dimer antennas.

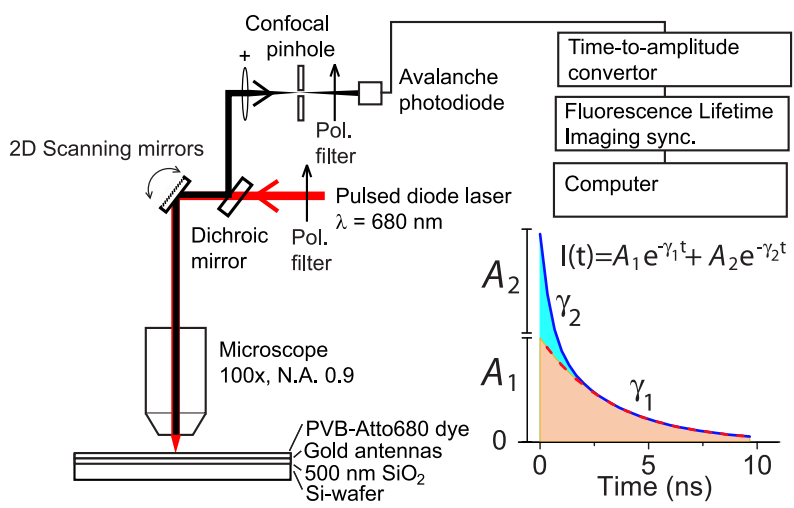

Fig. 2. Figure 2 Experimental setup for confocal fluorescence lifetime imaging, using a pulsed pump laser at $\lambda=660 \mathrm{~nm}$, a picosecond time-to-amplitude convertor (Picoquant) synchronized with a laser-scanning confocal microscope (Nikon). Inset: illustration of the double-exponential analysis of the time-resolved fluorescence decay, shown amplitudes $A_{1}, A_{2}$ and decay rates $\gamma_{1}, \gamma_{2}$, for the slow and fast contributions, respectively. 

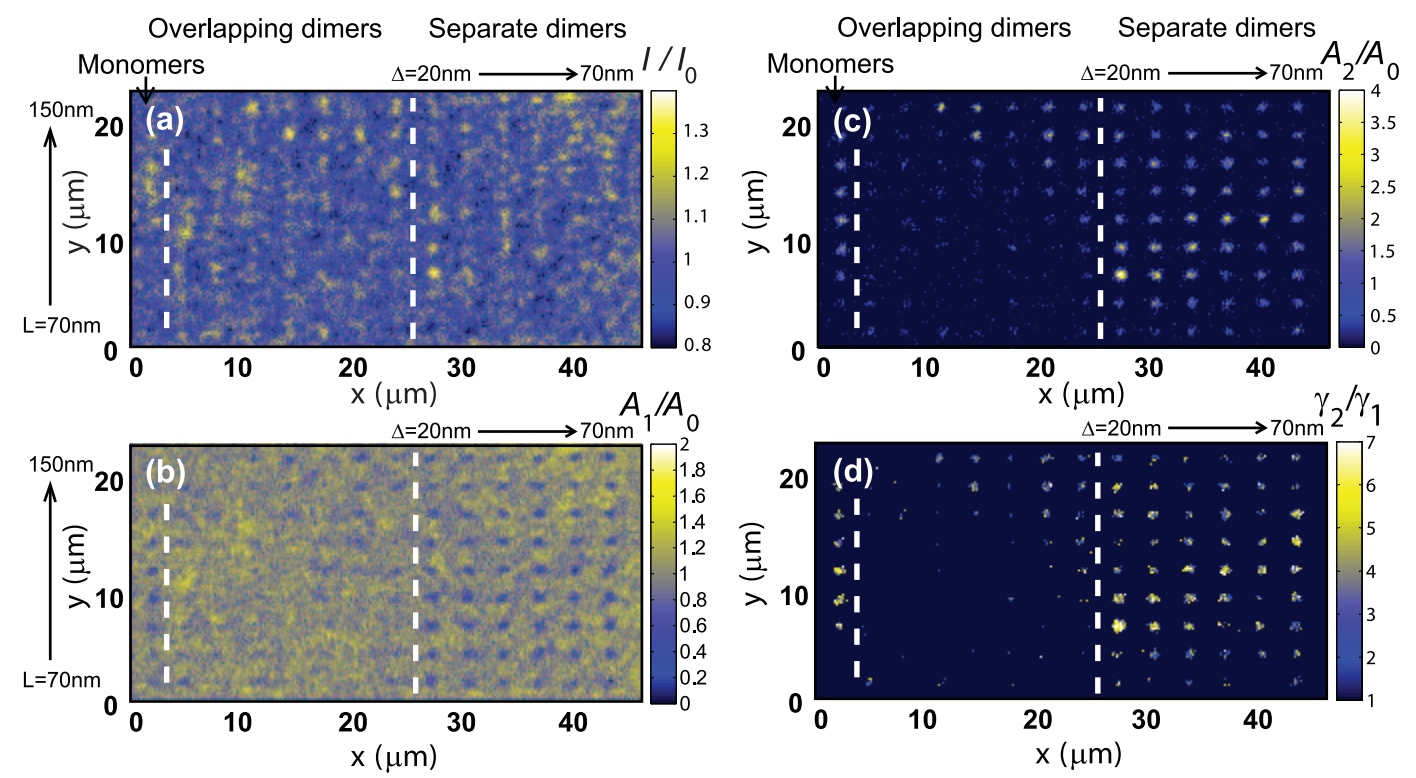

Fig. 3. Figure 3 Fluorescence lifetime image analysis of the nanoantenna array of Fig. 1(a), showing (a) total intensity $I$ normalized to average value $I_{0}$, (b) background amplitude $A_{1}$, and (c) fast component $A_{2}$, both normalized to average amplitude $A_{0}$. (d) Decay rate enhancement of the fast component. 